\title{
Orofacial functions and quality of life in children with unilateral cleft lip and palate
}

\author{
Ana Bheatriz Marangoni \\ MONTES(a) iD $^{(a)}$ \\ Thais Marchini OLIVEIRA(b) \\ Maria Beatriz Duarte GAVIÃO(a) ID \\ Taís de Souza BARBOSA(c) (iD \\ (a) Universidade Estadual de Campinas \\ - Unicamp, Piracicaba Dental School, \\ Department of Pediatric Dentistry, \\ Piracicaba, SP, Brazil. \\ (b) Universidade de São Paulo - USP, Bauru \\ School of Odontology, Department of \\ Pediatric Dentistry, Bauru, SP, Brasil. \\ (c) Universidade Federal de Juiz de Fora - \\ UFJF, Department of Dentistry, Governador \\ Valadares, MG, Brazil.
}

Declaration of Interests: The authors certify that they have no commercial or associative interest that represents a conflict of interest in connection with the manuscript.

\section{Corresponding Author:}

Prof. Taís de Souza Barbosa

E-mail: tais.barbosa@ufjf.edu.br

https://doi.org/10.1590/1807-3107bor-2019.vol33.0061

Submitted: 2017 August, 22

Accepted for publication: May 14, 2019

Last revision: June 10, 2019

\begin{abstract}
This study aimed to evaluate the orofacial functions and oral health-related quality of life (OHRQoL) of children with unilateral cleft lip and palate (UCLP). This case-control study included patients with UCLP matched by sex and age with controls (children without UCLP), resulting in the inclusion of a total of 108 eight- to ten-year-old children. Orofacial functions and OHRQoL were evaluated using the Nordic Orofacial Test-Screening (NOT-S) and the Child Perceptions Questionnaire $\left(\mathrm{CPQ}_{8-10}\right)$, respectively. Data normality was assessed by the Kolmogorov-Smirnov test. Differences and correlations in NOT-S and $\mathrm{CPQ}_{8-10}$ scores between and within the groups were evaluated using Mann-Whitney and Spearman's correlation tests, respectively. The distribution of NOT-S and global ratings of $\mathrm{CPQ}_{8-10}$ for each group were assessed by Chi-squared/Fisher's Exact tests. The UCLP group had a higher NOT-S total and examination scores than the controls. Dysfunctions related to breathing, facial symmetry/expression, and speech were more frequent in the UCLP patients than in the controls. The UCLP group had higher scores on the social well-being domain than the controls. There was a significant difference between the groups in their ratings in regards to the extent to which their oral condition affected their life overall, with controls perceiving it as somewhat better than patients. In both groups, NOT-S total and interview scores were positively correlated with $\mathrm{CPQ}_{8-10}$ total and domain scores. The NOT-S examination score was only significantly correlated with social domain scores in the control group. The presence of UCLP was associated with clinical signs of orofacial dysfunctions related to breathing, facial symmetry/expression, and speech. Children with UCLP reported more orofacial dysfunctions and negative impacts on social well-being than controls.
\end{abstract}

Keywords: Cleft Lip; Cleft Palate; Disability Evaluation; Facial Asymmetry; Quality of Life.

\section{Introduction}

Cleft lip and palate (CLP) is the second most common birth defect and the most common congenital craniofacial abnormality, affecting, on

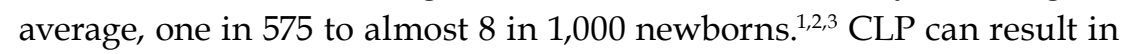
occlusal and anatomical alterations, most frequently a crossbite and skeletal 
class III malocclusion, ${ }^{4}$ and also seems to negatively impact the oral health-related quality of life (OHRQoL) of the affected children. ${ }^{5}$ Although demographic, socioeconomic, behavioral and psychosocial factors have been linked to OHRQoL, there is currently no agreement on the predictors in general and specific populations. ${ }^{6}$ Previous studies found a worse OHRQoL among Syrian ${ }^{7}$ and Brazilian children with CLP. Moreover, while some studies have suggested no differences for OHRQoL scores between children with or without CLP, ${ }^{9}$ others have reported lower negative impacts among CLP patients. ${ }^{10}$

Actually, the perceived OHRQoL measures may be considered as additional components to evaluate treatment of CLP patients in a more comprehensive way, considering that the assessment of outcomes frequently raises concerns about facial appearance, speech, and social impairments. ${ }^{11}$ In a recent systematic review and meta-analysis, the presence of CLP negatively affected the health-related quality of life (HRQoL) of children and adults, mainly on the psychological health and vitality dimensions. ${ }^{6}$ On the other hand, no significant differences were found considering oral health and oral conditions among the children in the evaluated studies. These results could be explained by the wide range of age of the subjects, which may have blended the different perceptions of OHRQoL among childhood and adolescence. ${ }^{12}$ Agespecific self-report measures are required to adapt differences in children's self-concepts, understanding of their feelings, and their ability to interpret other people's behavior. In terms of cognitive, emotional, social and language development, the age group of 8 to 10 years is fairly homogeneous. ${ }^{5}$ Despite this knowledge, there is a lack of studies about factors associated with the perception of OHRQoL related to CLP.

There are a number of distinct domains of OHRQoL measures involved in CLP children, such as appearance, speech, facial growth, and psychosocial interactions..$^{13}$ In the Leme et al. ${ }^{14}$ study, the presence of orofacial dysfunctions was associated with worse OHRQoL in otherwise healthy children, suggesting that oral and craniofacial diseases or disabilities may contribute to the compromise of vital actions (e.g., breathing, chewing, and swallowing) and muscle posture (e.g., mouth and tongue posture) and undermine the necessary requirements for social interactions, including speech, emotional communication, facial expression, and appearance. Notwithstanding, while there are existing studies evaluating the speech, muscle function, and facial asymmetry of children with CLP, $15,16,17,18$ there is no prior study correlating oral functions with impacts on OHRQoL. Considering the high prevalence of malocclusions among children with CLP, mainly a posterior crossbite that can result in facial asymmetry, abnormal tongue posture, an abnormal swallowing pattern, and temporomandibular disorders in adulthood, ${ }^{19,20}$ it is necessary to evaluate the orofacial functions of these patients as well as their correlations with OHRQoL.

Therefore, the present study aimed to evaluate the orofacial functions and OHRQoL of children with unilateral cleft lip and palate (UCLP). The research hypotheses were: a. orofacial dysfunctions affect children with UCLP and b. orofacial dysfunctions are correlated with OHRQoL.

\section{Methodology}

Ethical approval was obtained from the Human Research Ethics Committee of the Hospital for Rehabilitation of Craniofacial Anomalies (HRAC), University of São Paulo (USP), Bauru, SP, Brazil (protocol n. 318.871/2013). Participants and their parents were informed about the examination procedures and assured of the confidentiality of the collected information. Only those who signed the informed consent form were included in the study.

\section{Study Design and Sample Characteristics}

This case-control study was carried out among low-socioeconomic-status children aged 8 to 10 years. The children with UCLP (case group) were enrolled at HRAC/USP and the control group was represented by a population of children without CLP recruited from public schools of Piracicaba, SP, Brazil. The exclusion criteria for children with UCLP were tooth decay $(n=38)$, current use of an orthodontic appliance ( $n=74)$, syndromes associated with CLP (e.g., Treacher Collins, Van der Woude, Pierre Robin, Apert and 
others) $(n=14)^{21}$, and missing a consultation $(n=41)$. Exclusion criteria for the control group were tooth decay and current use of an orthodontic appliance. Thus, this study was conducted on 108 children matched by sex and age, resulting in 54 matched pairs. Data collection was conducted from July 2013 to November 2014.

\section{Sample Size Calculation}

The sample size was calculated on the basis of the NOT-S total score reported in a pilot study conducted with UCLP children enrolled in the HRAC/USP as described in our previous publication. ${ }^{22}$ Considering a mean of 0.24 NOTS total score, standard deviation of 0.9 , a sampling error of $10 \%$, and a confidence level of $95 \%$, the required sample size was defined as 54 individuals in each group.

\section{Orofacial Functions}

Orofacial functions were evaluated in the children with UCLP and the controls using the Brazilian Portuguese version ${ }^{14}$ of the Nordic Orofacial Test-Screening (NOT-S) protocol. ${ }^{23}$ This protocol consists of a structured interview and a clinical examination, each part including six domains. In the interview, the following functions were assessed: (I) sensory function, (II) breathing, (III) habits, (IV) chewing and swallowing, (V) drooling, and (IV) dryness of the mouth. In the examination, the following functions were assessed: (1) face at rest, (2) nose breathing, (3) facial expression, (4) masticatory muscle and jaw function, (5) oral motor function, and (6) speech. Each domain contains one to five items, reflecting the complexity of the specific function. The NOT-S was applied individually to each child by the same trained researcher (ABMM), in a vacant room, with the child seated in an upright position. The NOT-S interview was held by asking the questions printed on the screening form. To assess orofacial dysfunction in the clinical examination, the subjects were requested to carry out tasks for each item in conjunction with the illustrated manual. Each item has criteria for the respective function. An answer of YES or a task that met the criteria for impaired function resulted in a score of 1 , indicating a dysfunction in the scored domain. An answer of NO or a task that did not meet the criteria resulted in a score of 0 (zero). The total score was the sum of the score for each domain, ranging from 0 to 12 . The higher the score, the worse the orofacial dysfunction.

\section{Oral Health-Related Quality of Life}

The impact of oral conditions on the OHRQoL of children with UCLP and controls was evaluated using the Brazilian Portuguese version ${ }^{24}$ of the $\mathrm{CPQ}_{8-10 .}{ }^{5} \mathrm{It}$ is a self-completed questionnaire with 25 items grouped into four domains: oral symptoms, functional limitations, emotional well-being, and social well-being. A Likert-type scale was used with the response options of "Never" $=0$, "Once or twice" $=1$, "Sometimes" = 2, "Often" = 3, and "Very often" $=4$. The recall period was four weeks. The minimum possible score is zero and the maximum possible score is 100. A high score indicates more negative impacts on the OHRQoL.

Participants were also asked to give overall or global assessments of their oral health $(\mathrm{OH})$ and the extent to which the oral or orofacial condition affected their overall well-being (OWB). These questions preceded the multi-item scales in the questionnaire. $\mathrm{OH}$ and OWB questions are worded as follows: "When you think about your teeth or mouth, would you say that they are..." and "How much do your teeth or mouth bother you in your everyday life?" These global ratings had a 4-point response scale ranging from "Very good" $=0$ to "Poor"= 3 for $\mathrm{OH}$, and from "Not at all" $=0$ to "A lot" $=3$ for OWB, respectively.

\section{Statistical analysis}

Statistical analysis was performed using SPSS 22.0 (SPSS Inc., Chicago, IL, USA) and BioEstat 5.0 (Mamirauá, Belém, PA, Brazil) with a 5\% significance level. Data normality was assessed using the Kolmogorov-Smirnov test. Differences in NOT-S and $\mathrm{CPQ}_{8-10}$ scores between the UCLP and control groups were evaluated by means of the Mann-Whitney test. The magnitude of the difference between groups was assessed using the effect size (ES). This was derived from the mean difference in scores between the groups divided by the pooled SD of scores: a value of 0.2 was taken to be small, 0.5 to be moderate, and 0.8 to be large. ${ }^{25}$ 
The distribution of the NOT-S domain scores and responses to global ratings of $\mathrm{CPQ}_{8-10}$ for each group was assessed by Chi-squared or Fisher's Exact tests. The correlation between NOT-S and $\mathrm{CPQ}_{8-10}$ scores for each group was evaluated by Spearman's correlation test.

\section{Results}

This study evaluated orofacial functions and OHRQoL in 54 children with ULCP, matched by sex $(\hat{0}=57.41 \%)$ and age $(9.07 \pm 0.80$ years-old $)$ with controls. Table 1 shows the comparison of the NOT-S scores between the groups. The UCLP group had a higher mean NOT-S total score (4.3 vs. 2.8, $\mathrm{p}<0.0001)$ and examination ( 2.2 vs. $0.5, \mathrm{p}<0.0001)$ score than the controls.
The distribution of the NOT-S domains for each group are presented in Table 2. Children with UCLP reported more dysfunction related to breathing than controls $(29.6 \%$ vs. $7.4 \%$, p < 0.05$)$. All of these reports were related to snoring when sleeping. Clinical dysfunctions related to face at rest $(100 \%$ vs. $13 \%$; $\mathrm{p}<0.0001)$, facial expression (63\% vs. $22.2 \%, \mathrm{p}<0.01)$, and speech $(77.8 \%$ vs. $27.8 \%, \mathrm{p}<0.01)$ were more frequent among children with UCLP than the controls. Asymmetry (100\%) and deviant lip position (63\%) were the most frequent dysfunctions clinically diagnosed in patients when the face was at rest. More than half of the patients $(57.4 \%)$ could not pout or round their lips symmetrically (facial expression domain) and $74.1 \%$ presented with unclear speech in regards to one or more indistinct sounds or abnormal nasality.

Table 1. Comparison of the NOT-S scores between groups.

\begin{tabular}{lccc}
\hline \multirow{2}{*}{ Variable } & UCLP group & Control group & p-value* \\
\cline { 2 - 3 } & Mean (SD) & Mean (SD) & $<0.0001$ \\
Total scale [0-12] & $4.3(1.5)$ & $2.2(1.7)$ & 0.474 \\
Interview [0-6] & $1.6(1.2)$ & $1.7(1.3)$ & $<0.0001$ \\
Examination [0-6] & $2.8(0.9)$ & $0.5(0.7)$ & \\
\hline
\end{tabular}

NOT-S: nordic orofacil test-screning; UCLP: unilateral cleft lip and palate; sd: standard deviation. Values in square brackets indicate range of possible scores. ${ }^{*}$ p-value obtained from the Mann-Whitney test.

Table 2. Distribution [n (\%)] of the NOT-S domains by group.

\begin{tabular}{|c|c|c|c|}
\hline NOT-S domains & UCLP group & Control group & $p$-value \\
\hline \multicolumn{4}{|l|}{ Interview } \\
\hline (I) Sensory function & $4(7.4)$ & $5(9.3)$ & $1.000^{\mathrm{b}}$ \\
\hline (II) Breathing & $16(29.6)$ & $4(7.4)$ & $0.025^{a}$ \\
\hline (III) Habits & $17(31.5)$ & $35(64.8)$ & $0.058^{a}$ \\
\hline (IV) Chewing and swallowing & $38(70.4)$ & $34(63.0)$ & $0.831^{a}$ \\
\hline (V) Drooling & $2(3.7)$ & $2(3.7)$ & $1.000^{\mathrm{b}}$ \\
\hline (VI) Dry mouth & $7(13.0)$ & $12(22.2)$ & $0.418^{a}$ \\
\hline \multicolumn{4}{|l|}{ Examination } \\
\hline (1) Face at rest & $54(100.0)$ & $7(13.0)$ & $<0.0001^{\circ}$ \\
\hline (2) Nose breathing & $0(0.0)$ & $2(3.7)$ & $0.495^{b}$ \\
\hline (3) Facial expression & $34(63.0)$ & $12(22.2)$ & $0.010^{a}$ \\
\hline (4) Masticatory muscle and jaw function & $15(27.8)$ & $6(11.1)$ & $0.118^{a}$ \\
\hline (5) Oral motor function & $4(7.4)$ & $6(11.1)$ & $0.741^{b}$ \\
\hline (6) Speech & $42(77.8)$ & $15(27.8)$ & $0.005^{a}$ \\
\hline
\end{tabular}

NOT-S: nordic orofacil test-screning; UCLP: unilateral cleft lip and palate. ${ }^{\circ}$ Chi-squared test; ${ }^{b}$ Fisher's Exact test (comparison between groups) 
Table 3 shows the comparison of the $\mathrm{CPQ}_{8-10}$ scores between the groups. The mean overall scores for UCLP and the controls were 17.2 and 13.4 ( $p=0.132$ ), respectively. There was a significant difference between the groups only for the social well-being domain, with the UCLP group reporting, on average, more negative impacts than the controls (4.4 vs. 2.7, $\mathrm{p}<0.01$ ). However, the ES of 0.42 was only moderate and indicated that the difference between the groups was small in relation to the variability within the sample as a whole. Among the 10 items of social well-being domain, two were more reported by the UCLP group than the controls: being teased ( 0.9 vs. 0.2 ; $\mathrm{p}<0.01)$ or asked about their condition (1.4 vs. 0.3 ; $\mathrm{p}<0.001$ ) by other children.

Table 4 shows the distribution of the responses to the global ratings of the $C P Q_{8-10}$ in both groups. There was no difference between the two groups in their global ratings of $\mathrm{OH}$. The majority of children in the UCLP and control groups reported that the health of their teeth and mouth was OK $(37 \%)$ or very good $(57.4 \%)$, respectively. There was a significant difference between the two groups in their ratings of the extent to which their oral or orofacial condition affected their life overall, with the control group perceiving it as somewhat better than the UCLP patients ( 48.1 vs. $20.4 \%$; $p<0.05$ ). The majority of children in the UCLP and controls groups reported that their condition had little $(40.7 \%)$ or no effect (48.1\%) on their life overall.

Correlations between NOT-S and $\mathrm{CPQ}_{8-10}$ scores for each group are shown in Table 5. The NOT-S total scores were positively correlated with the $\mathrm{CPQ}_{8-10}$ total and subscales scores for both groups, except for the oral symptoms in the UCLP group. There were positive correlations between the NOT-S interview scores and the $\mathrm{CPQ}_{8-10}$ total and subscales scores for both groups. The NOT-S examination score was only significantly correlated with the social domain scores in the control group $(r=0.28, p<0.05)$.

Table 3. Comparison of $C P Q_{8-10}$ overall and domain scores between groups.

\begin{tabular}{lcccc}
\hline \multirow{2}{*}{$\begin{array}{l}\text { Variable } \\
\text { Overall scale }\end{array}$} & Range of possible scores & UCLP group & Control group & p-value \\
\cline { 3 - 4 } Oral symptoms & $0-100$ & Mean (SD) & Mean (SD) & 0.132 \\
Functional limitations & $0-20$ & $17.2(13.0)$ & $13.4(12.1)$ & 0.239 \\
Emotional well-being & $0-20$ & $6.0(2.8)$ & $5.2(3.8)$ & 0.060 \\
Social well-being & $0-20$ & $3.7(3.5)$ & $2.4(2.7)$ & 0.798 \\
\hline
\end{tabular}

CPQ: child perceptions questionnaire; UCLP: unilateral cleft lip and palate; SD: standard deviation. ${ }^{*} p$-value obtained from the Mann-Whitney test

Table 4. Distribution [n (\%)] of responses to global ratings by group.

\begin{tabular}{|c|c|c|c|}
\hline Variable & UCLP group & Control group & p-value* \\
\hline \multicolumn{4}{|c|}{ When you think about your teeth or mouth, would you say that they are: } \\
\hline Very good & $16(29.6)$ & $31(57.4)$ & $0.097^{a}$ \\
\hline Good & $16(29.6)$ & $12(22.2)$ & $0.644^{a}$ \\
\hline OK & $20(37.0)$ & $10(18.5)$ & $0.157^{a}$ \\
\hline Poor & $2(3.7)$ & $1(1.9)$ & $1.000^{\mathrm{b}}$ \\
\hline \multicolumn{4}{|c|}{ How much do your teeth or mouth bother you in your everyday life? } \\
\hline Not at all & $11(20.4)$ & $26(48.1)$ & $0.050^{\circ}$ \\
\hline A little bit & $22(40.7)$ & $15(27.8)$ & $0.421^{a}$ \\
\hline Some & $19(35.2)$ & $13(24.1)$ & $0.464^{a}$ \\
\hline A lot & $2(3.7)$ & $0(0.0)$ & $0.496^{b}$ \\
\hline
\end{tabular}

UCLP: unilateral cleft lip and palate. ${ }^{\circ} \mathrm{Chi}$-squared test: ${ }^{\text {bFisher's }}$ Exact test (comparison between groups) 
Table 5. Correlation between NOT-S and $C P Q_{8-10}$ scores for the UCLP group $(n=54)$.

\begin{tabular}{|c|c|c|c|}
\hline Variable & & VOT-S score & \\
\hline \multicolumn{4}{|l|}{ UCLP group } \\
\hline$C P Q_{8-10}$ overall scale & $038^{* *}$ & $0.47^{* * *}$ & 0.02 \\
\hline Oral symptoms & 0.25 & $0.35^{* *}$ & -0.05 \\
\hline Functional limitations & $0.35^{* *}$ & $0.47^{* * *}$ & -0.02 \\
\hline Emotional well-being & $0.37^{* *}$ & $0.38^{* *}$ & 0.11 \\
\hline Social well-being & $0.29^{*}$ & $0.35^{* *}$ & 0.02 \\
\hline \multicolumn{4}{|l|}{ Control group } \\
\hline$C P Q_{8-10}$ overall scale & $0.46^{\ldots *}$ & $0.47^{\cdots *}$ & 0.26 \\
\hline Oral symptoms & $0.37^{* *}$ & $0.38^{* *}$ & 0.14 \\
\hline Functional limitations & $0.41^{* *}$ & $0.44^{* * *}$ & 0.17 \\
\hline Emotional well-being & $0.32^{*}$ & $0.36^{* *}$ & 0.12 \\
\hline Social well-being & $0.37^{* *}$ & $0.35^{* *}$ & $0.28^{*}$ \\
\hline
\end{tabular}

NOT-S: nordic orofacil test-screning; UCLP: unilateral cleft lip and palate; CPQ: child perceptions questionnaire. " $p<0.05$; " $p<0.01$; $\cdots p<0.0001$ (Spearman correlation test).

\section{Discussion}

This study was undertaken to evaluate the orofacial functions and OHRQoL in 54 pairs of children with and without UCLP matched by sex and age, using NOT-S and $\mathrm{CPQ}_{8-10}$ measures, respectively. Both of the hypotheses tested were confirmed. That is, children with UCLP had poorer orofacial functions and more negative impacts on social well-being than controls. Further, the scores of both measures were correlated, suggesting that the more frequent their orofacial dysfunction, the greater the impairment of QoL in both populations. The strengths of this study include an a priori sample size calculation, as this is an essential item to reduce the probability of error ${ }^{26}$, and matching controls by age and sex, which allowed for the homogeneous distribution of these variables in the groups. Another strength of this investigation was the use of standardized questionnaires and also the standardized assessment of the oral conditions by a single calibrated/trained examiner.

In this present study, mean NOT-S total and examination scores were higher in the UCLP group than in the control group, suggesting more clinical signs of orofacial dysfunctions in the former, especially related to the face at rest, facial expression, and speech. In relation to the face at rest domain, all children with UCLP had skeletal and/or soft tissue asymmetry, while $63 \%$ had a deviant lip position when opening the mouth. Nasolabial asymmetry is a common finding in UCLP patients and in previous studies, using various methods to evaluate facial morphology, it has been shown that the cleft and non-cleft sides of the face differ. ${ }^{16}$

In relation to the facial expression domain, this domain measures the ability of the patient to pout and round the lips symmetrically, functions that depend on lip strength. In the present study, more than half of the patients (57.4\%) could not pout or round their lips symmetrically. This is contrary to Van Lierde et al.'s $\mathrm{s}^{18}$ findings of normal lip strength in children with unilateral UCLP. However, the comparison between the present findings and the above mentioned study should be interpreted with caution because they used different instruments: a screening instrument for clinical examination of orofacial dysfunction (NOT-S) and a hand-held device that measures the amount of pressure applied to a small pliable air-filled bulb in order to gage lip strength, tongue elevation strength, and tongue endurance (Iowa Oral Performance Instrument [IOPI]).

In relation to the speech domain, the majority of the patients in this study presented with unclear speech with one or more indistinct sounds or abnormal nasality. Speech and voice specialists state that because of the anatomical constraints present in UCLP infants, even the earliest attempts at vocalization in the second and third months of life in these children are clearly different from infants without a cleft defect. ${ }^{27}$ At about 6 months of age, a child without the defect 
starts to produce its first frontal consonants, whereas the structural deformity in a child with UCLP makes such a task impossible and may give rise to glottal or pharyngeal articulations.

Moreover, one third of the UCLP patients reported abnormal breathing in contrast with only $7.4 \%$ of the controls. All reports were related to snoring when sleeping. Children with UCLP have a higher incidence of obstructive sleep apnea, ${ }^{28}$ which is characterized by prolonged partial upper airway obstruction and/or an intermittent complete obstruction that disrupts both normal ventilation during sleep and normal neurophysiological sleep patterns. ${ }^{29}$ The size of their pharyngeal airways has been shown to be smaller and their craniofacial dimensions differ from those of healthy controls. ${ }^{29}$ The oropharyngeal musculature is also disrupted by the cleft, which impacts speech and swallowing, as well as adversely affecting the maintenance of airway patency, particularly during sleep.

The comparison between the present findings and other studies should be interpreted with caution because some studies used another OHRQoL questionnaire, different populations, and different methods. Broder and Wilson-Genderson ${ }^{30}$ reported that craniofacial patients had greater negative impacts on social-emotional well-being and school environment than general pediatric or orthodontic patients. Other studies using CPQ found no statistically significant difference between 8-10-yearold children with UCLP and children with dental caries $^{5}$ and healthy groups ${ }^{9}$ or found few differences in the OHRQoL of children aged 11 to 14 years with orofacial conditions compared with children with dental caries. Ward et al. ${ }^{10}$ found worse OHRQoL for the social-emotional well-being and functional well-being subscales among children with orofacial clefts compared with controls. In the present study, although the difference in the social well-being domain was statistically significant between the UCLP patients and controls, the ES suggested that the magnitude of the difference in the mean score for the two groups was moderate in relation to the variability among the study participants as a whole. Moreover, the mean difference between the groups was only 1.7 on a scale that could range from
0 to 40 . Additional evidence that the higher scores of the $\mathrm{CPQ}_{8-10}$ in the UCLP group may not be that important is to be found in the responses to the two global items. Overall, UCLP patients rated the health of their teeth and mouth similar to controls and frequently reported that their condition had little effect on their life overall (40.7\%). This leads to the conclusion that although the UCLP group may encounter more challenges in daily life, their overall QoL is no different from that of healthy children. This confirms the results of an earlier study that used $\mathrm{CPQ}_{11-14}$ to measure the outcomes of orofacial conditions and dental caries in children aged 11 to 14 years. ${ }^{31}$ The OHRQoL of the participants with cleft lip and/or palate was similar to that with dental decay. The present and above mentioned findings are consistent with the observation by Strauss ${ }^{32}$ in a literature review that, although appearance and speech may remain problematic for some people with orofacial conditions, the majority are "productive, contributing, happy, satisfied individuals."

The correlation analysis between the NOT-S and $\mathrm{CPQ}_{8-10}$ scores indicated an association between orofacial dysfunctions and worse OHRQoL in both groups in this study. However, clinical evidence of this relationship was observed only for the control group who presented a significant positive correlation between the NOT-S examination and the $\mathrm{CPQ}_{8-10}$ social domain scores. The patient-reported outcome measures would ascertain the patient's views of their symptoms, functional status, and HRQoL. However, assessing individuals with CLP can be difficult because congenital anomalies require long-term follow-up periods and serial assessment strategies to monitor treatment progress over time. ${ }^{33}$ In this context, our results may help in the understanding of how eight- to ten-year-old non-syndromic children with clefts perceive their health, guiding the professionals to provide a more holistic treatment. It is important to recognize the limitations of this work in terms of the methodology and analytic strategies used. The lack of temporality limits our confidence in establishing the direction of associations. Notwithstanding, cross-sectional investigations are important tools to identify risk indicators to be included in further longitudinal 
assessments. So, further research is needed to better explain the link between orofacial dysfunctions and OHRQoL in patients with UCLP.

\section{Conclusions}

Children with UCLP reported more orofacial dysfunctions and negative impacts on social well-being than controls. Moreover, the more frequent the orofacial dysfunction, the greater the impairment of QoL in both populations.

\section{Acknowledgments}

The authors gratefully acknowledge the financial support from the National Council for Scientific and Technological Development (CNPq, DF, Brazil, n. 140170/2014-6) and the volunteers for participating in this research.

\section{References}

1. Rodrigues K, Sena MF, Roncalli AG, Ferreira MA. Prevalence of orofacial clefts and social factors in Brazil. Braz Oral Res. 2009 Jan-Mar;23(1):38-42. https://doi.org/10.1590/S1806-83242009000100007

2. Matthews JL, Oddone-Paolucci E, Harrop RA. The Epidemiology of Cleft Lip and Palate in Canada, 1998 to 2007. Cleft Palate Craniofac J. 2015 Jul;52(4):417-24. https://doi.org/10.1597/14-047

3. World Health Organization. Global strategies to reduce the heath-care burden of craniofacial anomalies. Genebra: WHO; 2002.

4. Paradowska-Stolarz A, Kawala B. Occlusal disorders among patients with total clefts of lip, alveolar bone, and palate. BioMed Res Int. 2014;2014:583416. https://doi.org/10.1155/2014/583416

5. Jokovic A, Locker D, Tompson B, Guyatt G. Questionnaire for measuring oral health-related quality of life in eight- to ten-year-old children. Pediatr Dent. 2004 Nov-Dec;26(6):512-8.

6. Queiroz Herkrath AP, Herkrath FJ, Rebelo MA, Vettore MV. Measurement of health-related and oral health-related quality of life among individuals with nonsyndromic orofacial clefts: a systematic review and meta-analysis. Cleft Palate Craniofac J. 2015 Mar;52(2):157-72. https://doi.org/10.1597/13-104

7. Dak-Albab RJ, Dashash MA. The influence of socioeconomic status on oral health-related quality of life among Syrian children with cleft lip, or palate, or both. Saudi Med J. 2013 Feb;34(2):181-6.

8. Guedes RS, Piovesan C, Antunes JL, Mendes FM, Ardenghi TM. Assessing individual and neighborhood social factors in child oral healthrelated quality of life: a multilevel analysis. Qual Life Res. 2014 Nov;23(9):2521-30. https://doi.org/10.1007/s11136-014-0690-z

9. Wogelius P, Giørup H, Haubek D, Lopez R, Poulsen S. Development of Danish version of child oral-health-related quality of life questionnaires (CPQ8-10 and CPQ11-14). BMC Oral Health. 2009 Apr;9(1):11. https://doi.org/10.1186/1472-6831-9-11

10. Ward JA, Vig KW, Firestone AR, Mercado A, da Fonseca M, Johnston W. Oral health-related quality of life in children with orofacial clefts. Cleft Palate Craniofac J. 2013 Mar;50(2):174-81. https://doi.org/10.1597/11-055

11. Desmedt DJ, Maal TJ, Kuijpers MA, Bronkhorst EM, Kuijpers-Jagtman AM, Fudalej PS. Nasolabial symmetry and esthetics in cleft lip and palate: analysis of 3D facial images. Clin Oral Investig. 2015 Nov; 19(8):1833-42. https://doi.org/10.1007/s00784-015-1445-0

12. Barbosa TS, Gavião MB. Oral health-related quality of life in children: part II. Effects of clinical oral health status. A systematic review. Int J Dent Hyg. 2008 May;6(2):100-7. https://doi.org/10.1111/j.1601-5037.2008.00293.x

13. Klassen AF, Tsangaris E, Forrest CR, Wong KW, Pusic AL, Cano SJ, et al. Quality of life of children treated for cleft lip and/or palate: a systematic review. J Plast Reconstr Aesthet Surg. 2012 May;65(5):547-57. https://doi.org/10.1016/j.bjps.2011.11.004

14. Leme MS, Barbosa TS. Gavião MBD. Brazilian version of the Nordic Orofacial Test - Screening (NOT-S) for evaluation of orofacial dysfunctions. Pesqui Bras Odontopediatria Clin Integr. 2012;11(2):281-9. https://doi.org/10.4034/PBOCI.2011.112.21

15. Barsi PC, Ribeiro da Silva T, Costa B, da Silva Dalben G. Prevalence of oral habits in children with cleft lip and palate. Plast Surg Int. 2013;2013:247908. https://doi.org/10.1155/2013/247908

16. Fudalej P, Katsaros C, Hozyasz K, Borstlap WA, Kuijpers-Jagtman AM. Nasolabial symmetry and aesthetics in children with complete unilateral cleft lip and palate. Br J Oral Maxillofac Surg. 2012 Oct;50(7):621-5. https://doi.org/10.1016/i.bjoms.2011.11.019

17. Hortis-Dzierzbicka M, Radkowska E, Fudalej PS. Speech outcomes in 10-year-old children with complete unilateral cleft lip and palate after onestage lip and palate repair in the first year of life. J Plast Reconstr Aesthet Surg. 2012 Feb;65(2):175-81. https://doi.org/10.1016/i.bjps.2011.09.015

18. Van Lierde KM, Bettens K, Luyten A, Plettinck J, Bonte K, Vermeersch H, et al. Oral strength in subjects with a unilateral cleft lip and palate. Int J Pediatr Otorhinolaryngol. 2014 Aug;78(8):1306-10. https://doi.org/10.1016/i.ijporl.2014.05.017 
19. Melink S, Vagner MV, Hocevar-Boltezar I, Ovsenik M. Posterior crossbite in the deciduous dentition period, its relation with sucking habits, irregular orofacial functions, and otolaryngological findings. Am J Orthod Dentofacial Orthop. 2010 Jul;138(1):32-40. https://doi.org/10.1016/j.ajodo.2008.09.029

20. Tecco S, Festa F. Prevalence of signs and symptoms of temporomandibular disorders in children and adolescents with and without crossbites. World J Orthod. 2010;11(1):37-42.

21. Setó-Salvia N, Stanier P. Genetics of cleft lip and/or cleft palate: association with other common anomalies. Eur J Med Genet. 2014 Aug;57(8):381-93. https://doi.org/10.1016/i.ejmg.2014.04.003

22. Montes AB, Oliveira TM. Gavião MBD, Barbosa TS. Occlusal, chewing, and tasting characteristics associated with orofacial dysfunctions in children with unilateral cleft lip and palate: a case-control study. Clin Oral Investig. 2018 Mar;22(2):9421-50. https://doi.org/10.1007/s00784-017-2173-4

23. Bakke M, Bergendal B, McAllister A, Siögreen L, Asten P. Development and evaluation of a comprehensive screening for orofacial dysfunction. Swed Dent J. 2007;31(2):75-84.

24. Barbosa TS, Tureli MC, Gavião MB. Validity and reliability of the Child Perceptions Questionnaires applied in Brazilian children. BMC Oral Health. 2009 May;9(1):13. https://doi.org/10.1186/1472-6831-9-13

25. Cohen J. Statistical power analysis for the behavioural sciences. Hillside, NJ: Lawrence Erlbaum 1988.

26. Martínez-Mesa J, González-Chica DA, Bastos JL, Bonamigo RR, Duquia RP. Sample size: how many participants do I need in my research? An Bras Dermatol. 2014 Jul-Aug;89(4):609-15. https://doi.org/10.1590/abd1806-4841.20143705

27. O'Gara MM, Logemann JA, Rademaker AW. Phonetic features by babies with unilateral cleft lip and palate. Cleft Palate Craniofac J. 1994 Nov;31(6):446-51. https://doi.org/10.1597/1545-1569(1994)031<0446:PFBBWU>2.3.CO;2

28. Tan HL, Kheirandish-Gozal L, Abel F, Gozal D. Craniofacial syndromes and sleep-related breathing disorders. Sleep Med Rev. 2016 Jun;27:74-88. https://doi.org/10.1016/j.smrv.2015.05.010

29. Rose E, Thissen U, Otten JE, Jonas I. Cephalometric assessment of the posterior airway space in patients with cleft palate after palatoplasty. Cleft Palate Craniofac J. 2003 Sep;40(5):498-503. https://doi.org/10.1597/1545-1569_2003_040_0498_caotpa_2.0.co_2

30. Broder HL, Wilson-Genderson M. Reliability and convergent and discriminant validity of the Child Oral Health Impact Profile (COHIP Child's version). Community Dent Oral Epidemiol. 2007 Aug;35(1 Suppl 1):20-31. https://doi.org/10.1111/i.1600-0528.2007.0002.x

31. Locker D, Jokovic A, Tompson B. Health-related quality of life of children aged 11 to 14 years with orofacial conditions. Cleft Palate Craniofac J. 2005 May;42(3):260-6. https://doi.org/10.1597/03-077.1

32. Strauss RP. "Only skin deep": health, resilience, and craniofacial care. Cleft Palate Craniofac J. 2001 May;38(3):226-30. https://doi.org/10.1597/1545-1569_2001_038_0226_osdhra_2.0.co_2

33. Ranganathan K, Vercler CJ, Warschausky SA, MacEachern MP, Buchman SR, Waljee JF. Comparative effectiveness studies examining patient-reported outcomes among children with cleft lip and/or palate: a systematic review. Plast Reconstr Surg. 2015 Jan;135(1):198-211. https://doi.org/10.1097/PRS.0000000000000825 